\title{
Coopération entre FMH Inkasso Services SA et le bureau d'encaissement Encath SA à Bienne
}

\author{
Dr Jacques de Haller ${ }^{a}$, \\ Reinhard Kunz ${ }^{b}$ \\ a Président FMH Services \\ b Directeur FMH Services
}

Nous sommes heureux de vous informer de la nouvelle coopération de FMH Inkasso Services SA (FIS) avec le bureau d'encaissement Encath SA à Bienne (Inkas, centrale d'encaissement pour médecins et dentistes).

Cette coopération constitue l'un des pas décisifs dans la réorientation de FMH Services et de ses sociétés. FMH Inkasso Services SA est active depuis des décennies dans l'encaissement de créances pour les médecins praticiens. Ce marché s'est considérablement modifié ces dernières années et se caractérise actuellement par une concentration toujours plus forte. Dans le cadre de la réorganisation entreprise, nous nous sommes décidés de collaborer avec Inkas.

Cette nouvelle coopération apporte aux membres de la FMH des avantages importants. En tant que nouveau leader sur le marché des prestations d'encaissement, nous sommes à même de leur offrir dès le $1^{\mathrm{er}}$ mars 2006, au sein de la nouvelle entreprise, une gamme de produits et prestations des plus attrayants:

- collaborateurs de longue date, professionnalisme élevé et solide savoir-faire;

- nouveaux produits dotés d'avantages financiers certains pour le médecin;
- accès en ligne aux dossiers en cours;

- gestion des cas d'encaissement pratiquement sans documents papier.

D'ici à fin avril 2006, l'équipe actuelle de FIS transférera progressivement ses activités de Muri près de Berne à Bienne, en vue d'intégrer entièrement l'Inkas. La nouvelle firme offrira ses nouvelles prestations dès le $1^{\text {er }}$ mars 2006 à Bienne, alors que les dossiers en cours seront encore traités par FIS avec son infrastructure actuelle et aux conditions existantes. Le lieu de travail de Muri sera définitivement abandonné à fin avril 2006.

L'implication étroite de FMH Services dans la future conception et le marketing des produits destinés aux membres de la FMH permettra de garantir, dans le cadre d'une coopération fructueuse avec notre partenaire, des prestations de haute qualité avec un rapport prix/prestations au-dessus de la moyenne.

Avec cette nouvelle direction prise dans le domaine de l'encaissement, nous sommes convaincus de pouvoir apporter un soutien optimal aux médecins de la FMH en leur offrant des solutions particulièrement avantageuses en termes de coûts. 